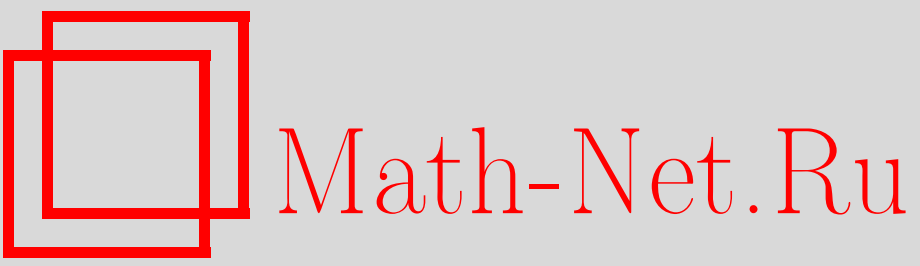

М. М. Глухов, О матрицах переходов разностей при использовании некоторых модулярных групп, Матем. вопр. криптогр., 2013, том 4, выпуск 4, 27-47

DOI: https://doi.org/10.4213/mvk98

Использование Общероссийского математического портала Math-Net.Ru подразумевает, что вы прочитали и согласны с пользовательским соглашением

http://www . mathnet.ru/rus/agreement

Параметры загрузки:

IP : 52.23 .180 .231

26 апреля 2023 г., 12:56:20 
УДК 512.542 .74

\title{
О матрицах переходов разностей при использовании некоторых модулярных групп
}

\author{
М. М. Глухов \\ Академия криптографии Российской Федерации, Москва \\ Получено 22.IV.2013 \\ В работе изучаются матрицы переходов биграмм для систем $G_{r} h G_{s}$ \\ подстановок степени $2^{n}$, где $G_{t}$ - группа сдвигов в прямой сумме групп \\ $\left(Z / 2^{t},+\right)$. Частично подтверждена известная гипотеза об отсутствии APN- \\ подстановок поля $G F\left(2^{n}\right)$ при четном $n$, предложены способы построения \\ Ключевые слова: модулярная группа, разностная характеристика сис-
} разностно-4-однородных подстановок. темы подстановок, $A P N$-функция

On the matrices of transitions of differences for some modular groups

M. M. Glukhov

Academy of Cryptography of the Russian Federation, Moscow

Abstract. Let $G_{t}$ be a translation group in a direct sum of groups $\left(Z / 2^{t},+\right)$. For the system of substitutions $G_{r} h G_{s}$ of order $2^{n}$ the matrices of digram transitions are investigated. A well-known hypothesis on the nonexistence of APN-substitutions of the field $\operatorname{GF}\left(2^{n}\right)$ for even $n$ is partly verified. Some methods of construction of differentially 4-uniform substitutions are suggested.

Key words: modular group, difference characteristics, systems of substitutions, APNfunctions

Citation: Mathematical Aspects of Cryptography, 2013, vol. 4, no. 4, pp. $27-47$ (Russian).

(C) 2013 Глухов М. М. 


\section{Введение}

Под модулярной группой будем понимать аддитивную группу прямой суммы любого числа экземпляров кольца вычетов $Z / m=\{0,1, \ldots, m-1\}$ по модулю $m>1$. Прямую сумму $k$ экземплляров кольца $Z / m$ будем обозначать $(Z / m)^{k}$. Здесь мы будем рассматривать такие группы лишь при условии, что $m$ есть степень числа 2 . Операции сложения и вычитания в кольце $Z / 2^{r}$ и в прямой сумме таких колец будем обозначать соответственно знаками $+_{r}$ и $-_{r}$ при $r>1$ и знаком $\oplus$ при $r=1$.

Зафиксируем целое число $n>0$, два делителя $2^{r}, 2^{s}$ числа $2^{n}$ и построим две модулярные группы $G_{r}=\left(Z / 2^{r}\right)^{n-r}, G_{s}=\left(Z / 2^{s}\right)^{n-s}$ порядка $2^{n}$. Записав элементы групп $Z / 2^{r}$ и $Z / 2^{s}$ в двоичной системе, представим элементы групп двоичными векторами длины $n$. Множество всех таких векторов обозначим через $V_{n}$. В конкретных случаях двоичный вектор $a=\left(a_{n-1}, \ldots, a_{1}, a_{0}\right)$ будем отождествлять с записью числа $a_{n-1} 2^{n-1}+\ldots+a_{1} 2+a_{0}$ в двоичной системе счисления или с набором коэффициентов элемента $a_{n-1} \theta^{n-1}+\ldots+a_{1} \theta+a_{0}$ поля $G F\left(2^{n}\right)$ при некотором примитивном элементе $\theta$. Всюду далее поле $G F\left(2^{n}\right)$ для краткости будем обозначать через $F_{n}$, а его мультипликативную группу через $F_{n}{ }^{*}$.

Таким образом, можно считать, что группы $G_{r}$ и $G_{s}$ определены на одном и том же множестве векторов $V_{n}$ и имеют один и тот же нулевой элемент $0=(0, \ldots, 0)$. Для сложения векторов $a, b \in V_{n}$ в группе $G_{r}\left(G_{s}\right)$ нужно их разбить на подвекторы длины $r(s)$ и, сложив соответствующие подвекторы как числа по модулю $2^{r}\left(2^{s}\right)$, записать результаты в виде двоичных векторов.

C точки зрения криптографических приложений представляет интерес задача изучения матриц переходов ненулевых разностей $G_{r}$ в разности элементов группы $G_{s}$ при различных $r, s$ под действием подстановок системы $G_{r} h G_{s}$ при некоторых подстановках $h$ из симметрической группы $S\left(V_{n}\right)$ всех подстановок множества $V_{n}$. Обозначим эту матрицу через $Q_{r, s}^{(n, h)}=\left(q_{a, b}^{(n, h, r, s)}\right), a, b \in V_{n}$. Ее элемент $q_{a, b}^{(n, h, r, s)}$ равен числу решений уравнения

$$
\left(a+{ }_{r} x\right) h-_{s} x h=b
$$

относительно неизвестного $x$, принимающего значения из $V_{n}$. 
В общем случае указанная задача является сложной. В данной работе мы ограничимся рассмотрением лишь некоторых частных случаев.

В большинстве общих рассуждений данной работы $n$ будет произвольным фиксированным целым числом и в индексах будет опускаться. С точки зрения криптографических приложений более интересным является случай, когда $n$ четно. Поэтому некоторые вопросы будут рассматриваться лишь при четном $n$. Во всех таких случаях будем считать, что $n=2 k>4$.

Максимальный элемент матрицы $Q_{r, s}^{(h)}$ назовем разностной характеристикой системы подстановок $G_{r} h G_{s}$ и обозначим через $\Delta\left(G_{r} h G_{s}\right)$. В случае $r=s=1$ эту величину будем обозначать через $\Delta(h)$. Если уравнение (1) имеет решения и один из элементов $a, b$ равен 0 , то и второй равен 0. При этом уравнению (1) удовлетворяет любой элемент из $V_{n}$. Поэтому далее будем предполагать, что $a \neq 0$ и $b \neq 0$. В связи с этим матрица $Q_{r, s}^{(n, h)}$ имеет размеры $\left(2^{n}-1\right) \times\left(2^{n}-1\right)$. При изучении матриц $Q_{r, s}^{(h)}=\left(q_{a, b}^{(h, r, s)}\right)$ будем сравнивать их с матрицей для системы подстановок $G_{1} h G_{1}$. Поэтому рассмотрим сначала матрицы $Q_{1,1}^{(h)}$.

\section{1. О свойствах матриц $Q_{1,1}^{(h)}$}

В этом крайнем частном случае для матрицы переходов ненулевых разностей введем особое обозначение $Q_{1,1}^{(h)}=P^{(h)}=\left(p_{a, b}^{(h)}\right)$. Ее элемент $p_{a, b}^{(h)}$ равен числу решений уравнения

$$
(a \oplus x) h \oplus x h=b .
$$

Приведем некоторые известные факты о матрицах $P^{(h)}$, включая для полноты чтения и их краткие доказательства.

1. Если $h-$ подстановка 2-го порядка, то матрица $P^{(h)}$ симметрична.

Действительно, уравнение (2) в этом случае можно преобразовать к виду $(b \oplus x h) h=a \oplus x$, и при замене переменных $x h=y$ получим уравнение

$$
(b \oplus y) h \oplus y h=a,
$$

имеющее столько же решений, сколько и уравнение (2). Следовательно, $p_{a, b}^{(h)}=p_{b, a}^{(h)}$.

2. При любой подстановке $h$ если $x_{1}-$ решение уравнения (2), то его решением является и $x_{2}=a \oplus x_{1}$.

Условимся решения $x_{1}$ и $a \oplus x_{1}$ уравнения (2) называть связанными. Таким образом, все решения уравнения (2) (если они существуют) разбиваются 
на пары связанных решений, причем очевидно, что разные связанные пары не пересекаются. Отсюда, в частности, получаем следующее свойство.

3. Все элементы матрицы $P^{(h)}$ четны, и потому число ненулевых элементов в каждой ее строке не превосходит $2^{n-1}$ (поскольку сумма элементов каждой ее строки равна $2^{n}$ ).

4. Если подстановка $g$ поля $F$ линейно эквивалентна подстановке $h$, т. е. $g=\varphi h \psi$ при некоторых $\varphi, \psi \in \operatorname{Aut}\left(F_{n}, \oplus\right)$, то матрица $P^{(g)}$ получается из матрицы $P^{(h)}$ перестановкой строк по подстановке $\varphi$ и столбцов по подстановке $\psi^{-1}$.

Действительно, уравнение $(a \oplus x) g \oplus x g=b$ преобразуется к виду

$$
\left(a_{1} \oplus y\right) h \oplus y h=b_{1} \text {, где } a_{1}=a \varphi, y=x \varphi, b_{1}=b \psi^{-1} .
$$

Следовательно, $p_{a, b}^{(g)}=p_{a_{1}, b_{1}}^{(h)}$, что и влечет свойство 4 .

Укажем еще свойства матриц $P^{(h)}$ при степенных подстановках $h$.

Утверждение 1. Пусть $m=2^{t} m_{1}>4,0 \leq t<n,\left(m_{1}, 2^{n}-1\right)=1 u h, h_{1}-$ степенные подстановки поля $F_{n}: h(x)=x^{m}, h_{1}(x)=x^{m_{1}}$. Тогда:

1) матрица $P^{\left(h_{1}\right)}$ отличается от матрицы $P^{(h)}$ лишь перестановкой строк;

2) все строки матрицы $P^{(h)}$ получаются перестановкой элементов одной ее строки.

Доказательство. Утверждение 1) есть частный случай свойства 4, поскольку возведение элементов поля $F_{n}$ во вторую степень является автоморфизмом группы $\left(F_{n}, \oplus\right)$.

Докажем утверждение 2). Пусть $a, b \in F_{n}{ }^{*}, p_{a, b}^{(h)}=c>0$ и $x_{1}$ - решение уравнения

$$
(a \oplus x)^{m} \oplus x^{m}=b .
$$

Уравнение (3) приводится к виду

$$
\sum_{i=1}^{m} C_{m}^{i} a^{i} x^{m-i}=b
$$

Так как $x_{1}$ - решение уравнения (4), то имеем равенство

$$
\sum_{i=1}^{m} C_{m}^{i} a^{i} x_{1}^{m-i}=b
$$


Любой элемент $a_{1} \in F_{n}^{*}$, отличный от 0 и $a$, можно представить в виде $a_{1}=a \theta^{r}$, где $\theta-$ примитивный элемент поля $F_{n}$ и $0<r<2^{n}-1$. Подставив в (5) $a=a_{1} \theta^{-r}$ и $x_{1}=x_{2} \theta^{-r}$, получим равенство

$$
\sum_{i=1}^{m} C_{m}^{i} a_{1}^{i} x_{2}^{m-i}=b \theta^{m r}
$$

которое означает, что $x_{2}=x_{1} \theta^{r}$ есть решение уравнения

$$
\sum_{i=1}^{m} C_{m}^{i} a_{1}^{i} x^{m-i}=b \theta^{m r}
$$

Так как неравенство $x_{1} \neq x_{1}^{\prime}$ влечет $x_{1} \theta^{r} \neq x_{1}^{\prime} \theta^{r}$, то имеем: $p_{a_{1}, b \theta^{m r}}^{(h)} \geq p_{a, b}^{(h)}$. И это неравенство имеет место при любом $b$, при котором $p_{a, b}^{(h)}>0$. Теперь учитывая, что сумма всех элементов по строкам матрицы одна и та же, получаем $p_{a_{1}, b \theta^{n r}}^{(h)}=p_{a, b}^{(h)}$, и значит, состав элементов в строках с номерами $a$ и $a_{1}$ один и тот же, т. е. любая строка матрицы $P^{(h)}$ получается перестановкой элементов строки с номером $a$, и утверждение 2 ) верно. В итоге утверждение 1 полностью доказано.

Далее линейно эквивалентные подстановки будем называть просто эквивалентными.

Из свойства 4 следует, что для эквивалентных подстановок $g, h$ выполняется равенство

$$
\Delta\left(G_{1} g G_{1}\right)=\Delta\left(G_{1} h G_{1}\right) .
$$

В силу утверждения 1) далее при рассмотрении степенных подстановок $h(x)=x^{m}$, не теряя общности, можно считать, что $m$ нечетно.

\section{2. О системах подстановок с разностной характеристикой 2}

Строение матрицы $P^{(h)}$ полностью определяется подстановкой $h$ и изучалось при различных подстановках $h$ многими авторами. Известны примеры подстановок, при которых $\Delta(h)=2$ и $\Delta(h)=2^{n-2}$. Для подстановки $h$, выбираемой случайно и равновероятно из симметрической группы подстановок пространства $V_{n}$, В. Н. Сачков [1] указал интервал, содержащий максимальный элемент матрицы переходных вероятностей ненулевых разностей с вероятностью, стремящейся к 1 при $n \rightarrow \infty$, и улучшил асимптотическую оценку математического ожидания величины $\Delta(h)$ из [10]. 
Для криптографических приложений наибольший интерес представляют подстановки с минимальным значением величины $\Delta(h)$, которое равно 2. Заметим, что отображения $h: V_{n} \rightarrow V_{n}$, при которых уравнение (2) имеет ровно 2 решения при любых $a, b \in V_{n}$, в работе [7] были названы почти совершенно нелинейными функциями, короче, $A P N$-функциями. К настоящему времени имеется большой цикл работ, посвященных поиску $A P N$-функций, в основном, для случая, когда $V_{n}$ совпадает с аддитивной группой поля $F_{n}$. Построен ряд степенных $A P N$-функций и $A P N$-функций, не эквивалентных степенным функциям (краткий обзор работ по APN-функциям см. в [3]). Далее нас будут интересовать лишь функции $h: V_{n} \rightarrow V_{n}$, являющиеся подстановками. Такие $A P N$-функции будем называть $A P N$-подстановками. К сожалению, ни одна из найденных до сих пор $A P N$-функций не является подстановкой поля $F_{n}$ при четном $n$, и, как отмечено в [5], известные специалисты (Cantenaut, Carlet, Sharpin, Dobbertin, Zinoviev) считают справедливой гипотезу об отсутствии $A P N$-подстановок поля $F_{n}$ при четном $n$.

Частичным подтверждением этой гипотезы является следующее утверждение. Заметим предварительно, что каждая подстановка поля $F_{n}$ представляется многочленом степени, меньшей $2^{n}$ над полем $F_{n}$.

Утверждение 2. Если п четно $u$ h- подстановка поля $F_{n}$, представимая многочленом

$$
h(x)=a_{m} x^{m} \oplus \ldots \oplus a_{1} x \oplus a_{0}, a_{m} \neq 0,
$$

в котором сумма коэффициентов при степенях $x$, кратных трем, равна 0 , то $h$ не является $A P N$-подстановкой.

Доказательство. Пусть $h \in S\left(V_{n}\right)$. Рассмотрим сначала случай, когда $h$ - степенная подстановка, т. е. $h(x)=x^{m},\left(m, 2^{n}-1\right)=1$, и возведение в степень производится в поле $F$. Из доказательства утверждения 1 следует, что, не теряя общности, можно считать число $m$ нечетным. Для доказательства утверждения 2 в рассматриваемом случае достаточно доказать существование таких $a, b \in V_{n}$, при которых уравнение (2) имеет более двух решений. Пусть $a=b=1$. Тогда уравнение (2) запишется в виде

$$
(x \oplus 1)^{m} \oplus x^{m}=1 .
$$

Очевидно, что ему удовлетворяют 0 и 1 . Покажем, что его корнями являются также все элементы порядка 3 поля $F_{n}$. Так как $n$ четно, то $2^{n}-1$ кратно 3 , и элементами порядка 3 являются лишь два элемента $\alpha=\theta^{d}$ и $\alpha^{2}=\theta^{2 d}$, где $\theta-$ примитивный элемент поля $F_{n}$ и $d=\left(2^{n}-1\right) / 3$. Заме- 
тим, что $0,1, \alpha, \alpha^{2}$ образуют подполе порядка 4 поля $F$, и в нем $\alpha \oplus \alpha^{2}=1$. Подставим $\alpha$ вместо $x$ в уравнение (7) и докажем, что верно равенство

$$
(\alpha \oplus 1)^{m} \oplus \alpha^{m}=1 .
$$

Так как $\left(m, 2^{n}-1\right)=1$, то $m$ не кратно трем, и значит, $m \equiv 1(\bmod 3)$ или $m \equiv 2(\bmod 3)$.

Пусть $m \equiv 1(\bmod 3)$. Так как $\alpha \oplus 1=\alpha^{2}$, то равенство (8) можно записать в виде $\alpha^{2 m} \oplus \alpha^{m}=1$. Так как $m \equiv 1(\bmod 3)$, то $\alpha^{m}=\alpha, \alpha^{2 m}=\alpha^{2}$, и равенство (8) следует из равенства $\alpha \oplus \alpha^{2}=1$. В случае $m \equiv 2(\bmod 3)$ равенство (8) доказывается аналогично. Этим утверждение для степенных подстановок доказано. Очевидно, что тогда оно верно и для подстановок $h(x)=c x^{m}$ при любом $c \in F_{n} \backslash\{0\}$.

Пусть теперь $h$ - любая подстановка поля $F_{n}$, представленная многочленом (6) над $F_{n}$. Тогда уравнение (2) запишется в следующем виде:

$$
\left(a_{m}(a \oplus x)^{m} \oplus a_{m} x^{m}\right) \oplus \ldots \oplus\left(a_{1}(a \oplus x) \oplus a_{1} x\right)=b .
$$

Положим $a=1$, а $b$ равным сумме всех коэффициентов многочлена $h(x)$, отличных от $a_{0}$. Заметим, что $b \neq 0$, так как в противном случае многочлен $h(x)$ принимал бы значение $a_{0}$ при $x=0$ и $x=1$, т. е. не был бы подстановочным. Учитывая условие на коэффициенты многочлена $h$, paвенство (8) при $(\mathrm{m}, 3)=1$ и равенство $A_{l}=a_{l}(1 \oplus \alpha)^{l} \oplus a_{l} \alpha^{l}=0$ при $l$, кратном трем, нетрудно убедиться в том, что уравнению (9) удовлетворяют все элементы $0,1, \alpha, \alpha^{2}$. Следовательно, подстановка $h$ не является $A P N$-подстановкой, и утверждение 2 доказано.

\section{3. О системах подстановок с разностной характеристикой 4}

В связи с отсутствием примеров $A P N$-подстановок поля $F_{n}$ при четном $n$ представляют интерес подстановки $h$, при которых максимальный элемент матрицы $P^{(h)}$ равен 4 , т. е. $\Delta(h)=4$.

В работе [8] для конечных абелевых групп $G, G^{\prime}$ отображение $h: G \rightarrow G^{\prime}$ названо разностно- $\delta$-однороднымм, если $\Delta\left(G h G^{\prime}\right) \leq \delta$.

К разностно-4-однородным подстановкам группы $\left(F_{n}, \oplus\right)$ при четных $n>4$ относятся все подстановки, эквивалентные подстановке $\tau$ обращения ненулевых элементов поля, доопределенной равенством $0 \tau=0$. Матрица 
переходов разностей при действии системы подстановок $G_{1} \tau G_{1}$ хорошо известна. Подстановку $\tau$ при любом $n$ впервые рассмотрел Б.А. Егоров в 1969 году. Им было замечено, что она при любом $n>4$ лучше других известных подстановок $h$ с точки зрения близости матрицы $P^{(h)}$ к равномерной матрице. Из его результатов следовало, что при нечетном $n$ подстановка $\tau$ является $A P N$-функцией, а при четном $n$ в каждой строке матрицы $P^{(\tau)}$ находится $2^{n-1}$ нулей, $2^{n-1}-2$ двоек и лишь одна четверка. Отметим, что система подстановок $G_{1} \tau G_{1}$ при $n=8$ использована в шифрсистеме AES.

Укажем еще некоторые свойства матрицы $P^{(\tau)}$. Предварительно заметим, что аддитивная группа поля $F_{n}$ имеет $2^{n}-1$ максимальных подгрупп, и любая из них имеет вид $a H$, где $a \in F_{n}{ }^{*}, H=\left\{c \in F_{n}: \operatorname{tr}(c)=0\right\}, \operatorname{tr}-$ функция-след из поля $F_{n}$ в поле $G F(2)$. Очевидно, что $H$ - группа по сложению.

Утверждение 3. Если $n$ четно, то в строке с номером $a \neq 0$ матриизы $P^{(\tau)}$ ненулевые элементы находятся в столбиах, номера которых образуют множество элементов, обратных $\kappa$ ненулевым элементам подгруппы $а H$.

Доказательство. Пусть $p_{a, b} \neq 0$, т. е. уравнение

$$
(a \oplus x) \tau \oplus x \tau=b
$$

имеет решения. Если среди решений есть 0 , а значит, и $a$, то $a \tau=b$, т. е. $b=a^{-1}$. Следовательно, в этом случае $b \in(a H)^{-1}$, и утверждение верно. Если же среди решений есть элементы, отличные от 0 и $a$, то уравнение (10) с помощью подстановки $x=a y$ преобразуется к уравнению

$$
y^{2} \oplus y \oplus 1 / a b=0 .
$$

Известно (см., например, [2], стр. 32 ), что уравнение (11) имеет решение в том и только том случае, когда $(1 / a b) \in H$, т. е. $b \in(a H)^{-1}$. Следовательно, и в этом случае утверждение верно.

Замечание. Доказательство утверждения 3 приведено здесь лишь для полноты чтения. В действительности, оно является следствием формул для элементов $p_{a, b}^{(\tau)}$ матрицы $P^{(\tau)}$, доказанных Б. В. Березиным (1984 г.):

$$
\begin{gathered}
p_{a, b}=0, \text { если } \operatorname{tr}(1 / a b)=1, \\
p_{a, b}=2, \text { если } \operatorname{tr}(1 / a b)=0, b \neq a^{-1},
\end{gathered}
$$




$$
p_{a, b}=4, \text { если } \operatorname{tr}(1 / a b)=0, b=a^{-1} .
$$

Следствие. При любом $n>1$ матрииа $\left(P^{(\tau)}\right)^{2}$ не имеет нулевых элементов, т. е. множество подстановок $G_{1} \tau G_{1} \tau G_{1}$ дважды транзитивно.

Доказательство. Так как $\tau$ - подстановка 2-го порядка, то матрица $P^{(\tau)}$ симметрична, и в силу утверждения 3 при четном $n$ ненулевые элементы столбца с номером $b$ матрицы $P^{(\tau)}$ находятся в строках, номера которых являются элементами, обратными к элементам подгруппы $b H$. Теперь наше утверждение при четном $n$ следует из очевидного соотношения $a H \cap b H \neq \varnothing$ при любых $a, b \in F_{n}^{*}$. Для нечетного $n$ утверждение следствия выводится непосредственно из правила умножения матриц, если учесть, что в этом случае в каждой строке и в каждом столбце матрицы $P^{(\tau)}$ содержится более половины ненулевых элементов.

Б. В. Березин (1984 г.) доказал также, что система подстановок $G_{1} \tau$ при $n>4$ порождает знакопеременную группу подстановок поля $F_{n}$.

Из приведенных свойств подстановки $\tau$ следует, что система подстановок $G_{1} \tau G_{1}$ обладает хорошим перемешивающим свойством.

Отметим и некоторые недостатки подстановки $\tau$ при ее использовании в боксах шифрсистем. Так как $\tau-$ подстановка 2-го порядка, то матрица переходов разностей для системы $G_{1} \tau G_{1}$ при одинаковой нумерации ее строк и столбцов симметрична и четверки в ней расположены по некоторой побочной диагонали. Отсюда, в частности, следует, что в квадрате этой матрицы диагональные элементы равны $2^{n+1}+16$, а каждый из остальных элементов равен $2^{n}$ или $2^{n}+4$. И эта неравномерность довольно долго сохраняется при возведении матрицы в степени $3,4, \ldots$.

Кроме того, при действии подстановки $\tau$ имеется достаточно простая связь $x y=1$ выхода $y$ со входом $x$, что облегчает применение $X S L$-метода анализа соответствующих блочных шифров.

Свойство матрицы $P^{(\tau)}$, указанное в утверждении 3 , также является определенным минусом для подстановки $\tau$, поскольку легко позволяет находить расположение ненулевых элементов каждой строки матрицы $P^{(\tau)}$.

В связи с этим многими авторами осуществлялся поиск других подстановок поля $F_{n}$, обладающих хорошими криптографическими качествами. Каждая подстановка поля $F_{n}$ реализуется многочленом. Существует большое число работ, посвященных описанию подстановочных многочленов над полем $F_{n}$. 
Ряд результатов в этом направлении был получен Е. А. Тришиным. Так, например, в его диссертации доказан критерий подстановочности для некоторых многочленов вида $x^{r} g\left(x^{t}\right)$ над полем $G F(q)$, где $t$ - делитель $q-1$. Среди них при $q=2^{8}$ экспериментальным путем найдено много подстановок $h$, для которых $\Delta(h)=4$.

C точки зрения реализации подстановок наибольший интерес представляют многочлены небольших степеней. Приведем известные результаты о подстановочных многочленах степеней $m \leq 7$ над полем $F_{n}$ при четном $n$. В случае существования многочлены $h(x)$ степени $m$ приводятся обычно в нормализованном виде, т. е. унитарные, с условием $h(0)=0$ и с нулевым вторым коэффициентом, если $(m, 2)=1$. Полный список всех нормализованных подстановочных многочленов при $m \leq 5$ приведен в [4], а при $m \leq 6$ в [7].

Для подстановочных многочленов $h(x)$ степеней 1,2 степень многочлена $h(x \oplus a) \oplus h(x)$ равна 0, и потому $\Delta(h)$ принимает максимально возможное значение $2^{n}$.

Подстановочных многочленов степеней 3 и 6 над полем $F_{n}$ при четном $n$ не существует.

Все нормализованные подстановочные многочлены степени 4 исчерпываются многочленами вида $h(x)=x^{4} \oplus b x^{2} \oplus c x$. Для них ситуация та же, что и при степенях 1,2 , поскольку здесь $h(x \oplus a) \oplus h(x)=a^{4} \oplus b a^{2} \oplus a$ не зависит от $x$.

При $n=5$ единственным нормализованным подстановочным многочленом над полем $F_{n}$ является $h(x)=x^{5}$ и только при условии $\left(2^{n}-1,5\right)=1$. В этом случае уравнение (2) имеет вид $a x^{4} \oplus a^{4} x \oplus a^{5}=b$ и, очевидно, имеет не более 4 корней при любых $a, b \in F_{n}^{*}$. Отсюда, учитывая свойство 3 (см. п. 1) матрицы $P^{(h)}$ и утверждение 2 , получаем $\Delta(h)=4$. (Если $2^{n}-1$ кратно 5 , то отображение $h(x)=x^{5}$ не является подстановкой по критерию Диксона, см. [4], с. 440).

И. В. Бобылев (1985 г.) доказал, что при $n=7$ существует лишь два класса линейно-эквивалентных подстановочных многочленов, их представителями являются многочлены $x^{7}, x^{7} \oplus x^{5} \oplus x$.

В общем случае, когда подстановка $h$ задается многочленом степени $k$ над $F_{n}$, выполняется неравенство $\Delta(h) \leq k-1$, поскольку степень многочлена $h(x \oplus a) \oplus h(x)$ не превосходит $k-1$ и потому уравнение (2) 
имеет не более $k-1$ решений при любом $b \in F^{*}$. Примерами, когда $\Delta(h)=k-1$, могут служить подстановки $h(x)=x^{7}$ и $h(x)=x^{7} \oplus x^{5} \oplus x$ поля $G F\left(2^{8}\right)$. Компьютерными вычислениями для подстановки $h(x)=x^{7}$ поля $F_{8}$, являющегося основным полем шифрсистемы $A E S$ (т. е. расширением поля $G F(2)$ с помощью корней многочлена $\left.f(x)=x^{8} \oplus x^{4} \oplus x^{3} \oplus x^{2} \oplus 1\right)$, установлено, что в этом случае $\Delta(h)=6$.

Укажем еще один способ построения разностно-4-однородных подстановок на подмножествах множества $V_{n}$ при четных $n$. Предварительно введем новое обозначение и докажем вспомогательное утверждение.

Обозначим через $Q_{0}^{(m, h,+)}$ матрицу переходов всех разностей (включая и нулевую) абелевой группы $\left(V_{m},+\right)$ под действием системы подстановок $\gamma_{x} h \gamma_{y}$, где $a \gamma_{x}=a+x$, а $h$ - подстановка на $V_{m}$.

Утверждение 4. Пусть абелева группа $\left(V_{n},+\right)$ есть внешняя прямая сумма групп $\left(V_{r}, *\right),\left(V_{t}, \circ\right), h_{1} \in S\left(V_{r}\right), h_{2} \in S\left(V_{t}\right), u h=\left(h_{1}, h_{2}\right)$ - такая подстановка из симметрической группь $S\left(V_{n}\right)$, что $(a, b) h=\left(a h_{1}, b h_{2}\right)$ для любых $a \in V_{r}, b \in V_{t}$. Тогда существует нумерачия строк и столбцов матриць $Q_{0}^{(n, h,+)}$, при которой

$$
Q_{0}^{(n, h,+)}=Q_{0}^{\left(r, h_{1}, *\right)} \otimes Q_{0}^{\left(t, h_{2}, \circ\right)},
$$

где $\otimes-$ - знак тензорного произведения матриц.

Доказательство. Упорядочим произвольно элементы множеств $V_{r}$ и $V_{t}$. Пусть

$$
\alpha_{1}<\alpha_{2}<\ldots<\alpha_{2^{r}} \text { в } V_{r} \text { и } \beta_{1}<\beta_{2}<\ldots<\beta_{2^{t}} \text { в } V_{t} .
$$

Теперь упорядочим элементы из $V_{n}$ лексикографически, т. е. положим

$$
\left.\left(\alpha_{i}, \beta_{j}\right)<\left(\alpha_{k}, \beta_{l}\right) \Leftrightarrow \alpha_{i}<\alpha_{k} \text { или } \alpha_{i}=\alpha_{k}, \beta_{j}<\beta_{l}\right) .
$$

Будем считать, что строки и столбцы матрицы $Q_{0}^{(m, h,+)}$ занумерованы элементами множества $V_{n}$ по их возрастанию в указанном выше порядке. По определению матрицы $Q_{0}^{(m, h,+)}$ ее элемент из строки с номером $\left(\alpha_{i}, \beta_{j}\right)$ и столбца с номером $\left(\alpha_{k}, \beta_{l}\right)$ равен числу решений уравнения

$$
\left((x, y)+\left(\alpha_{i}, \beta_{j}\right)\right) h-(x, y) h=\left(\alpha_{k}, \beta_{l}\right),
$$


которое в силу условий леммы равносильно системе уравнений

$$
\begin{aligned}
& \left(x * \alpha_{i}\right) h_{1} * x h_{1}=\alpha_{k}, \\
& \left(y \circ \beta_{j}\right) h_{2} \circ y h_{2}=\beta_{l},
\end{aligned}
$$

где $\bar{*}, \overline{0}$ - операции, обратные соответственно к операциям $*$, . Так как неизвестные $x, y$ независимы, то число решений этой системы равно произведению чисел решений входящих в нее уравнений. Следовательно, указанный элемент матрицы $Q_{0}^{(m, h,+)}$ равен произведению элементов с мест $\left(\left(\alpha_{i}, \alpha_{k}\right),\left(\beta_{j}, \beta_{l}\right)\right)$ соответственно матриц $Q_{0}^{\left(r, h_{1}, *\right)}, Q_{0}^{\left(t, h_{2}, \circ\right)}$. Значит, при фиксированных $\alpha_{i}, \alpha_{k}$ элементы матрицы $Q_{0}^{(m, h,+)}$ с мест $\left(\left(\alpha_{i}, \beta_{j}\right),\left(\alpha_{k}, \beta_{l}\right)\right)$ при всевозможных $\beta_{j}, \beta_{l}$ образуют подматрицу, равную произведению элемента матрицы $Q_{0}^{\left(r, h_{1}, *\right)}$ с номером $\left(\alpha_{i}, \alpha_{k}\right)$ на матрицу $Q_{0}^{\left(t, h_{2}, \text { o) }\right.}$. Это с учетом выбранной нумерации строк и столбцов матриц свидетельствует о выполнении равенства (12), и утверждение доказано.

Следствие 1. Если в утверждении 4 операщии $+, *, \circ$ совпадают с операцией $\oplus, n=r+s$, где $r, s$ нечетны, и $h_{1}, h_{2}-A P N$-подстановки, то подстановка $h$ является разностно-4-однородной на множестве $\left(V_{t} \backslash\{0\}\right) \times\left(V_{r} \backslash\{0\}\right)$.

Замечание. Подстановки из следствия 1 могут использоваться в блочных шифрах типа $X S L$, если $h$ оставляет на месте нулевой элемент, a $L-$ невырожденное линейное преобразование пространства $V_{n}$ над полем $G F(2)$.

\section{4. О свойствах матриц $Q_{2,1}^{(h)}$ и $Q_{1,2}^{(h)}$}

Пусть $Z / 4=\{0,1,2,3\}$ - кольцо вычетов по модулю 4. В соответствии с введенными обозначениями операцию сложения в этом кольце будем обозначать чере $t_{2}$, а числа $0,1,2,3$ записывать в двоичной системе счисления: $0=(0,0), 1=(0,1), 2=(1,0), 3=(1,1)$. В общем виде $x=\left(x_{1}, x_{0}\right)$. Тогда элемент прямой суммы $k$ экземпляров кольца $Z / 4$ запишется в виде двоичного вектора длины $2 k$. Как и выше, будем считать $2 k=n$ и операцию сложения в $(Z / 4)^{k}$ обозначать знаком $+_{2}$. Для простоты прямую сумму $k$ экземпляров группы $\left(Z / 4,+_{2}\right)$ обозначим буквой $G$. Так как в данном пункте индексы 2,1 у матрицы $Q_{2,1}^{(h)}$ будут фиксированными, то матрицу $Q_{2,1}^{(h)}$, ее строку с номером $a$ и ее элемент $q_{a, b}^{(h, 2,1)}$ будем обозначать соответ- 
ственно $Q^{(h)}, Q_{a}^{(h)}$ и $q_{a, b}^{(h)}$. Отметим, что элемент $q_{a, b}^{(h)}$ равен числу решений уравнения

$$
\left(a+{ }_{2} x\right) h \oplus x h=b .
$$

Выше отмечалось, что в каждой строке матрицы $P^{(h)}$ число ненулевых элементов не превосходит $2^{n-1}$. Представляет интерес вопрос о сравнении матрицы $Q^{(h)}$ с матрицей $P^{(h)}$. С этой целью воспользуемся связью между операциями $+_{2}$ и $\oplus$.

Утверждение 5. Если $a=\left(a_{1}, a_{0}\right), b=\left(b_{1}, b_{0}\right) \in Z / 4$, то

$$
\begin{aligned}
& a+{ }_{2} b=\left(a_{1} \oplus b_{1} \oplus a_{0} b_{0}, a_{0} \oplus b_{0}\right), \\
& a-{ }_{2} b=\left(a_{1} \oplus b_{1} \oplus a_{0} b_{0} \oplus b_{0}, a_{0} \oplus b_{0}\right) .
\end{aligned}
$$

Утверждение доказывается непосредственной проверкой.

Из утверждения 5 получаем два следствия.

Следствие 1. Пусть $a=\left(a_{n-1}, \ldots, a_{1}, a_{0}\right), b=\left(b_{n-1}, \ldots, b_{1}, b_{0}\right)$ - элементы из G. Тогда

$$
\begin{aligned}
& a+{ }_{2} b=a \oplus b \Leftrightarrow a_{0} b_{0}=a_{2} b_{2}=\ldots=a_{n-2} b_{n-2}=0, \\
& a-{ }_{2} b=a \oplus b \Leftrightarrow \bar{a}_{0} b_{0}=\bar{a}_{2} b_{2}=\ldots=\bar{a}_{n-2} b_{n-2}=0,
\end{aligned}
$$

где $\bar{a}_{i}=a_{i} \oplus 1$ - отрииание элемента $a_{i}, i=0,2, \ldots, n-2$.

Следствие 2. Для любых элементов

$$
a=\left(a_{n-1}, \ldots, a_{1}, a_{0}\right), b=\left(b_{n-1}, \ldots, b_{1}, b_{0}\right) \in V_{n}
$$

выполняются равенства

$$
a+{ }_{2} b=a \oplus b \oplus c, a-{ }_{2} b=a \oplus b \oplus d,
$$

где $c=\left(c_{n-1}, \ldots, c_{1}, c_{0}\right), d=\left(d_{n-1}, \ldots, d_{1}, d_{0}\right)$,

$$
c_{i}=\left\{\begin{array}{ccc}
0, & \text { если } i \text { четно } \\
a_{i-1} b_{i-1}, & \text { если } i \text { нечетно }
\end{array}, d_{i}=\left\{\begin{array}{ccc}
0, & \text { если } i \text { четно } \\
\left(a_{i-1} \oplus 1\right) b_{i-1}, & \text { если } i \text { нечетно }
\end{array} .\right.\right.
$$

Так как элементы $c, d$ из утверждения 5 зависят лишь от $a$ и $b$, то их естественно обозначить $c=c_{a, b}, d=d_{a, b}$. Тогда равенства следствия 2 принимают вид

$$
a+_{2} b=a \oplus b \oplus c_{a, b}, \quad a-{ }_{2} b=a \oplus b \oplus d_{a, b} .
$$

Введем еще для $a=\left(a_{n-1}, \ldots, a_{1}, a_{0}\right)$ обозначения:

$$
\delta_{0}(a)=\left(a_{n-2}, a_{n-4}, \ldots, a_{0}\right), \delta_{1}(a)=\left(a_{n-1}, a_{n-3}, \ldots, a_{1}\right) .
$$


Рассмотрим вопрос о решении уравнения (13). В зависимости от элемента $\delta_{0}(a)$ здесь возможны различные ситуации. Рассмотрим сначала два простейших случая, когда

$$
a=\left(a_{n-1}, \ldots, a_{1}, a_{0}\right),\left\|\delta_{0}(a)\right\|=0 \text { или }\left\|\delta_{0}(a)\right\|=1 .
$$

Число таких значений $a$, что $\left\|\delta_{0}(a)\right\|=0$, равно $2^{n / 2}$. По следствию 1 из утверждения 5 тогда $a \oplus_{2} x=a \oplus x$, и потому множество решений уравнения (13) совпадает с множеством решений уравнения (2), т. е. $q_{a, b}^{(h)}=p_{a, b}^{(h)}$ при любом $b$. Следовательно, строка $Q_{a}^{(h)}$ матрицы $Q^{(h)}$ совпадает со строкой $P_{a}^{(h)}$ матрицы $P^{(h)}$. Таким образом, в рассматриваемом случае $2^{n / 2}$ строк матрицы $Q^{(h)}$ совпадают с соответствующими строками матрицы $P^{(h)}$.

Пусть $\left\|\delta_{0}(a)\right\|=1$ и $a_{2 i}=1$ при некотором $i \in\{0,1, \ldots,(n-2) / 2\}$. В этом случае все значения $x=\left(x_{n-1}, \ldots, x_{1}, x_{0}\right)$ разобьем на два подмножества:

$$
\left\{x \mid x_{2 i}=0\right\},\left\{x \mid x_{2 i}=1\right\},
$$

и в соответствии с этим уравнение (13) будет равносильным объединению двух систем уравнений

$$
\text { 1) }\left\{\begin{array}{l}
\left(\left(a+{ }_{2} x\right) h \oplus x h=b,\right. \\
x_{2 i}=0,
\end{array} \text { и } 2\right)\left\{\begin{array}{l}
\left(\left(a+{ }_{2} x\right) h \oplus x h=b,\right. \\
x_{2 i}=1 .
\end{array}\right.
$$

В системе 1) согласно (14) $a+{ }_{2} x=a \oplus x$, и ее первое уравнение совпадает с уравнением (2). Если уравнение (2) имеет решения, то каждая пара его связанных решений имеет вид $\left(x^{\prime}, x^{\prime \prime}=a \oplus x^{\prime}\right)$. Так как $a_{2 i}=1$, то только одно из решений $x^{\prime}, x^{\prime \prime}$ удовлетворяет условию $x_{2 i}=0$. Следовательно, система 1) имеет $\frac{1}{2} p_{a, b}^{(h)}$ решений. В системе 2) $a+{ }_{2} x=a \oplus x \oplus 2^{2 i}$, и ее первое уравнение принимает вид

$$
\left(\left(a \oplus 2^{2 i}\right) \oplus x\right) h \oplus x h=b .
$$

Если это уравнение не имеет решений, то и система 2) не имеет решений. Если же оно имеет решения, то в решениях из одной связанной пары компоненты с номером $2 i$ совпадают, и потому оба решения одновременно либо не удовлетворяют, либо удовлетворяют условию $x_{2 i}=1$. В первом случае система 2) не имеет решений, во втором имеет $p_{a \oplus 2 i, b}^{(h)}$ решений. 
В итоге

$$
\text { либо } q_{a, b}^{(h)}=\frac{1}{2} p_{a, b}^{(h)} \text {, либо } q_{a, b}^{(h)}=\frac{1}{2} p_{a, b}^{(h)}+p_{a \oplus 2 i, b}^{(h)} \text {. }
$$

Теперь рассмотрим случай, когда

$$
a=\left(a_{n-1}, \ldots, a_{1}, a_{0}\right) \text { и }\left\|\delta_{0}(a)\right\|=s>1 .
$$

Пусть $a_{2 i_{1}}=a_{2 i_{2}}=\ldots=a_{2 i_{s}}=1$, а остальные компоненты вектора $a$ равны нулю. Тогда уравнение (13) распадется на $2^{s}$ систем уравнений вида

$$
\left\{\begin{array}{l}
\left(\left(c_{j_{1}, \ldots, j_{t}} \oplus x\right) h \oplus x h=b,\right. \\
x_{2 j_{1}}=\ldots=x_{2 j_{t}}=1, x_{2 j}=0: \forall j \in\left\{i_{1}, \ldots, i_{s}\right\} \backslash\left\{j_{1}, \ldots, j_{t}\right\},
\end{array}\right.
$$

где $0 \leq t \leq s,\left\{j_{1}, \ldots, j_{t}\right\}$ - подмножество множества $\left\{i_{1}, i_{2}, \ldots, i_{s}\right\}$, которое считаем пустым при $t=0$, а вектор $c_{j_{1}, \ldots, j_{t}}$ получен заменой в $a$ единичных компонент $a_{2 j_{1}}, \ldots, a_{2 j_{t}}$ нулями.

Множество решений системы (15) обозначим через $K_{j_{1}, \ldots, j_{t}}$. Так как при заданных значениях $b, x$ коэффициент $c_{j_{1}, \ldots, j_{t}}$ находится однозначно из первого уравнения системы (15), то при различных наборах $\left\{j_{1}, \ldots, j_{t}\right\}$ соответствующие множества $K_{j_{1}, \ldots, j_{t}}$ не пересекаются.

Для решения системы (15) решим сначала ее первое уравнение. Возможны 3 варианта: а) решений нет, б) решения есть, но они не содержатся в $K_{j_{1}, \ldots, j_{t}}$, в) решения есть, и часть из них содержится в $K_{j_{1}, \ldots, j_{t}}$. В случаях a), б) система (15) не имеет решений. Рассмотрим случай в). Если $t<s$ и $x=u$ - решение первого уравнения, содержащееся в $K_{j_{1}, \ldots, j_{t}}$, то связанное с ним решение $u \oplus c_{j_{1}, \ldots, j_{t}}$ не может принадлежать $K_{j_{1}, \ldots, j_{t}}$. Если же $t=s$, то в паре связанных решений первого уравнения системы (15) либо оба элемента не содержатся в $K_{j_{1}, \ldots, j_{t}}$, либо оба содержатся в $K_{j_{1}, \ldots, j_{t}}$. Соответствующие последнему случаю наборы $\left\{j_{1}, \ldots, j_{t}\right\}$ назовем особыми, а их множество обозначим через $K_{0}$.

Из проведенных рассуждений видно, что в общем случае получить точную формулу, выражающую элемент $q_{a, b}^{(h)}$ через элементы матрицы $P^{(h)}$, вряд ли возможно, поскольку значение величины $q_{a, b}^{(h)}$ зависит не только от числа решений уравнений вида (2) при различных значениях $a=c_{j_{1}, \ldots, j_{t}}$, но 
и от самих решений этих уравнений, существенно зависящих от подстановки $h$. Поэтому точные значения величины $\Delta\left(G_{2} h G_{1}\right)$ можно находить только при конкретных значениях $n$ и $h$. В общем же случае из проведенных рассуждений получаются лишь следующие оценки.

Утверждение 6. Для любого $n$ и любой подстановки $h$ поля $F_{n}$

$$
\Delta\left(G_{2} h G_{1}\right) \leq \max _{s}\left(\frac{1}{2} \sum_{J \notin K_{0}} p_{c_{J}, b}^{(h)}+\sum_{J_{0} \in K_{0}} p_{c_{J_{0}}, b}^{(h)}\right),
$$

где $J_{0}$ пробегает все особые наборы, $J-$ все остальные из указанных выше наборов при фиксированном значении $s$, а максимум берется по всем $s \in\left\{1,2, \ldots, 2^{n-1}\right\}$.

Замечание. Оценка (16) является весьма грубой. Если предположить, что решения первого уравнения в (15) образуют случайную и равновероятную выборку из поля $F_{n}$, то каждое такое решение будет удовлетворять остальным уравнениям с вероятностью $1 / 2^{s}$. Следовательно, в среднем верхняя оценка не должна превосходить величины

$$
\max _{s} \frac{1}{2^{s}}\left(\frac{1}{2} \sum_{J \notin K_{0}} p_{c_{J}, b}^{(h)}+\sum_{J_{0} \in K_{0}} p_{c_{J_{0}}, b}^{(h)}\right) .
$$

Эксперименты с рядом конкретных подстановок при $n=8$ показывают, что величина $\Delta\left(G_{2} h G_{1}\right)$ близка к $\Delta\left(G_{1} h G_{1}\right)$. Так, например, для подстановок $h(x)=x^{7}$ и $h=\tau$ поля $G F\left(2^{8}\right)$, используемого в $A E S$, выполняется равенство $\Delta\left(G_{2} h G_{1}\right)=\Delta\left(G_{1} h G_{1}\right)+1$.

Сравним теперь матрицы $Q_{2,1}^{(h)}$ и $Q_{1,2}^{(h)}$.

Утверждение 7. Для любого $n$, любой подстановки $h$ поля $F_{n} u$ для любых $a, b \in F_{n}^{*}$ выполняется равенство $q_{a, b}^{(h, 2,1)}=q_{b, a}^{\left(h^{-1}, 1,2\right)}$.

Доказательство. Элемент $q_{a, b}^{(h, 2,1)}$ есть число решений уравнения

$$
\left(a+{ }_{2} x\right) h \oplus x h=b,
$$

которое можно привести к виду $(b \oplus x h) h^{-1}-{ }_{2} x=a$. Произведя в нем замену $x=y h^{-1}$, получим уравнение относительно $y$

$$
(b \oplus y) h^{-1}-{ }_{2} y h^{-1}=a,
$$

имеющее столько же решений, сколько и уравнение (17). Это и доказывает утверждение 7. 
Следствие. Для любого $n$ и любой подстановки $h$ поля $F_{n}$

$$
\Delta\left(G_{1} h G_{2}\right)=\Delta\left(G_{2} h G_{1}\right) .
$$

Замечание. При изучении матрицы $Q_{2,2}^{(h)}$ понадобится решать уравнения вида

$$
\left(a+{ }_{2} x\right) h-{ }_{2} x h=b .
$$

Решение этого уравнения при $\left\|\delta_{0}(a)\right\|=k$ сведется к решению $2^{k}$ систем уравнений (17) точно так же, как уравнение (13) сводилось к системам уравнений вида (2). В матрице $Q_{2,2}^{(h)}$ число строк, совпадающих со строками матрицы $P^{(h)}$, будет в общем случае значительно меньше, чем в матрице $Q_{2,1}^{(h)}$, поскольку для указанного совпадения, кроме условия $\left\|\delta_{0}(a)\right\|=0$, необходимо еще условие $\left\|\delta_{0}\left(\left(a+{ }_{2} x\right) h\right)\right\|=0$. В этом случае найти точное значение или хорошие оценки для величины $\Delta\left(G_{2} h G_{2}\right)$ еще более затруднительно.

\section{5. О некоторых системах подстановок типа $G_{n} h G_{1}$}

Приведем еще серию подстановок вида $G_{n} h_{m} G_{1}$ с разностной характеристикой $\Delta\left(G_{n} h_{k} G_{1}\right) \leq 4$.

Зафиксируем произвольно примитивный корень $\theta$ поля $F_{n}$ и для каждого из чисел $m=1,2, \ldots, 2^{n}-2$ определим подстановку $h_{m}$, положив $0 h_{m}=0$ и $x h_{m}=\theta^{x+m}$ при $x \in\left(Z / 2^{n}\right)^{*}$.

Утверждение 8. Для любой из определенных выме подстановок $h_{m}$ выполняются неравенства $3 \leq \Delta\left(G_{n} h_{m} G_{1}\right) \leq 4$.

Доказательство. Непосредственной проверкой можно убедиться в том, что если $x \neq 0, a+_{n} x \neq 0$ и $a>1$, то

$$
\Delta_{a}=\left(a+{ }_{n} x\right) h_{m} \oplus x h_{m}=\theta^{x+m}\left(\theta^{a} \oplus 1\right) \text { при } x=1,2, \ldots, 2^{n}-a-1,
$$

$$
\Delta_{a}=\left(a+{ }_{n} x\right) h_{m} \oplus x h_{m}=\theta^{x+m}\left(\theta^{a-1} \oplus 1\right) \text { при } x=2^{n}-a+1,2^{n}-a+2, \ldots, 2^{n}-1 .(19
$$

При $a=1$ множества значений $x$ и $\Delta_{a}$ из (19) являются пустыми. Если $x=0$ или $x=-{ }_{n} a$, то $\Delta_{a}$ равно соответственно $\theta^{m+a}$ и $\theta^{m-a+1}$. 
Очевидно, что при любом $a$ все значения разности $\Delta_{a}$ из (18) различны, и то же самое верно для значений разности $\Delta_{a}$ из (19). Таким образом, в любом случае $\Delta\left(G_{n} h_{m} G_{1}\right) \leq 4$. Покажем, что $\Delta\left(G_{n} h_{m} G_{1}\right) \geq 3$. Легко видеть, что равенство $\theta^{m-a+1}=\theta^{m+a}$ выполняется лишь при $a=2^{n-1}$, и значит, при $a \neq 2^{n-1}$ элементы $\theta^{m+a}$ и $\theta^{m-a+1}$ различны. Теперь покажем, что найдется такое $a \neq 2^{n-1}$, при котором элементы $\theta^{m+a}$ и $\theta^{m-a+1}$ не содержатся среди значений $\Delta_{a}$ из (18). Допустим, что

$$
\theta^{x+m}\left(\theta^{a} \oplus 1\right)=\theta^{a+m} .
$$

Отсюда при $\theta^{x} \neq 1$, т. е. при $x \neq 0,2^{n}-1$, находим

$$
\theta^{a}=\frac{1}{\left(1 \oplus 1 / \theta^{x}\right)}
$$

Правая часть этого равенства принимает $2^{n}-2$ различных значений из $F_{n}^{*}$, когда $x$ пробегает значения $1,2, \ldots, 2^{n}-2$. Поэтому, выбрав любое значение $x$ из (19), отличное от $2^{n}-1$, мы из (20) найдем $a$, при котором элемент $\theta^{m+a}$ не содержится среди разностей из (18). Число указанных значений $x$, а потому и соответствующих значений $a$, равно $\left(2^{n}-1\right)-\left(2^{n}-a\right)-1=a-2$. Заметим еще, что равенство (20) при $x=2^{n}-1$ не выполняется. Таким образом, найдется $a-1$ значений $a$ при которых $\theta^{m+a}$ не содержится среди разностей из (18).

Аналогично доказывается, что число значений $a$, при которых элемент $\theta^{m-a+1}$ не содержится среди разностей из (18), также равно $a-1$. Следовательно, найдется значение $a>3$, при котором элементы $\theta^{m+a}$ и $\theta^{m-a+1}$ различны и не содержатся среди разностей из (18) и (19). Следовательно, $\Delta\left(G_{n} h_{k} G_{1}\right) \geq 3$, и утверждение доказано.

Замечание. Система подстановок $G_{n} h_{m} G_{1}$ при $m=0$ исследовалась в [5], в частности, в этом случае указаны условия, при которых $\Delta\left(G_{n} h_{0} G_{1}\right)=3$.

Для сравнения систем подстановок типа $G_{r} h G_{s}$ с точки зрения их перемешивающих свойств приведем таблицу некоторых параметров для систем подстановок поля $G F\left(2^{8}\right)$, используемого в $A E S$, при $h_{1}=\tau$ и $h_{2}(x)=x^{7}$. 


\section{Таблица 1}

$\begin{array}{lcccc}G_{1} h_{1} G_{1} & 4 & 128 & (256,528) & 272 \\ G_{2} h_{1} G_{1} & 5 & 82 & (194,370) & 176 \\ G_{4} h_{1} G_{1} & 7 & 85 & (203,338) & 135 \\ G_{8} h_{1} G_{1} & 6 & 87 & (199,317) & 118 \\ G_{4} h_{1} G_{4} & 8 & 93 & (196,554) & 358 \\ G_{8} h_{1} G_{8} & 6 & 93 & (191,566) & 375 \\ G_{1} h_{2} G_{1} & 6 & 156 & (176,536) & 360 \\ G_{2} h_{2} G_{1} & 7 & 100 & (170,398) & 228 \\ G_{4} h_{2} G_{1} & 7 & 95 & (185,360) & 175 \\ G_{8} h_{2} G_{1} & 7 & 94 & (186,346) & 180 \\ G_{4} h_{2} G_{4} & 7 & 94 & (185,327) & 142 \\ G_{8} h_{2} G_{8} & 7 & 93 & (194,322) & 128\end{array}$

Таблица 1 содержит 5 столбцов. В 1-м указана система подстановок $G_{r} h_{i} G_{s}$, во 2-м - максимальный элемент матрицы $Q_{r, s}^{(h)}$, в 3-м - среднее число нулей по строкам матрицы $Q_{r, s}^{(h)}$, в 4-м - минимальный и максимальный элементы матрицы $\left(Q_{r, s}^{(h)}\right)^{2}$, в 5-м - разность элементов из столбца 4.

Из таблицы 1 видно, что с точки зрения числа ненулевых элементов по строкам и разности между максимальным и минимальным элементами квадратов матриц системы $G_{2} h G_{1}$ значительно лучше соответствующих систем $G_{1} h G_{1}$ и для $h=\tau$, и для $h(x)=x^{7}$. Максимальный элемент матриц в последних случаях на единицу больше, однако встечается он в матрицах весьма редко. Так, например, в матрице для системы $G_{2} \tau G_{1}$ максимальный элемент 5 встречается лишь 3 раза.

Сравним характер связей между входами и выходами в системах $G_{1} h G_{1}$ и $G_{2} h G_{1}$. В первом случае на вход подстановки $h$ поступает элемент $a \oplus x$, и если $y$ - выход, то связь между $x$ и $y$ выражается равенством $x \oplus y h^{-1}=a$. Во втором случае на вход поступает элемент $a+{ }_{2} x$, и связь между $x$ и $y$ выражается равенством $x \oplus c_{a, x} \oplus y h^{-1}=a$, которое в отличие от первого случая содержит функцию $c_{a, x}$ (см. (14)). В частности, при $h=\tau$ очень простое соотношение $(a \oplus x) y=1$ в первом случае заменится более сложным во втором. Заметим, что матрица $Q^{(\tau)}$ в отличие от матрицы $P^{(\tau)}$ 
не симметрична и в ней не наблюдается каких либо структурных особенностей. Строки в матрице $\left(Q^{(\tau)}\right)^{2}$, как и в матрице $\left(P^{(\tau)}\right)^{2}$, не содержат нулей.

Сделаем одно замечание. Линейная характеристика подстановки $h$ при ее использовании в системе $G_{1} h G_{1}$ обычно определяется как

$$
L_{h}=\max _{a, b}|\{x:(a, h(x))=(b, x)\}|,
$$

где $a=\left(a_{n-1}, \ldots, a_{1}, a_{0}\right), b=\left(b_{n-1}, \ldots, b_{1}, b_{0}\right), x=\left(x_{n-1}, \ldots, x_{1}, x_{0}\right)$ - двоичные записи элементов $a, b, x, h(x)=\left(h_{n-1}(x), \ldots, h_{1}(x), h_{0}(x)\right)$ - координатная форма подстановки $h$, а под $(\cdot, \cdot)$ понимается скалярное произведение векторов над полем $G F(2)$ в ортонормированном базисе.

Так как в системе $G_{2} h G_{1}$ после подстановки $h$ применяется операция $\oplus$, то при использовании подстановки $h$ в системе $G_{2} h G_{1}$ в качестве линейной характеристики естественно принять ту же самую величину $L_{h}$.

В заключение приведем для наглядности одну строку матрицы $G_{2} \tau G_{1}$. 1320200000100102130020123111021210111111112 20222112002111120000100101110110102322012011 21212011010121001220120131001010223101203311 10021200020011013122002211101012110120200010 30200011013121222101020113100211121102012210 200100201211301010220112211121001121

Из приведенных результатов о системе подстановок $G_{2} h G_{1}$ следует, что она, допуская реализацию примерно той же сложности, что и система $G_{1} h G_{1}$, обладает по сравнению с ней лучшими криптографическими качествами.

В заключение выражаю признательность рецензенту данной работы В. А. Шишкину за ряд ценных замечаний.

\section{Список литературы}

1. Сачков, В. Н. Цепи Маркова итерационных систем преобразований // В сб.: Труды по дискретной математике, т. 6. - М.: ФИЗМАТЛИТ, 2002. - C. $165-183$.

2. Степанов, С. А. Арифметика алгебраических кривых. - М.: Наука, 1991.

3. Тужсилин, М. Э. Почти совершенные нелинейные функции // Прикладная дискретная математика. - 2009. - № 3. - С. 14-20.

4. Лидл, Р., Нидеррайтер, Г. Конечные поля, т. 2. - М.: Мир, 1988. 
5. Agievich, C., Afonenko, A. On the properties of exponential substitutions // Proc. Nat. Acad. Sci. of the Republic of Belarus. Physics and Math. Ser. 2005. - № 1. - P. 106-112.

6. Budaghyan, L., Carlet, C., Leandr, G. A class of quadratic APN binomials inequivalent to power functions // Cryptology ePrint Archive, 2006/428.

7. Dickson, L. E. The analytic representation of substitutions on a power of a prime number of letters with a discussion of the linear group // Ann. Math. - 1897. - V. 11. № 1/6. - P. 65-120, 161-183.

8. Nyberg, K., Knudsen, L. R. Provable security against differential cryptanalysis // CRYPTO'92. Lect. Notes Comput. Sci. — 1993. - V. 740. — P. 566574.

9. Nyberg, $K$. Differentially uniform mappings for cryptography // EUROCRYPT'93. Lect. Notes Comput. Sci. - 1994. - V. 765. - P. 55-64.

10. Hawkes, $P$., O'Connor, L. XOR and non-XOR differential probabilities // EUROCRYPT'99. Lect. Notes Comput. Sci. - 1999. - V. 1592. - P. 272285 . 
